\title{
Delivery of bevacizumab by intracranial injection: assessment in glioma model
}

This article was published in the following Dove Press journal: OncoTargets and Therapy

\author{
Yu-Xiao Liu',* \\ Wen-Jia Liü,* \\ Hui-Ru Zhang ${ }^{1,3}$ \\ Zhi-Wen Zhang' \\ 'Department of Neurosurgery, \\ First Affiliated Hospital of Chinese \\ PLA General Hospital, Beijing, \\ People's Republic of China; ${ }^{2}$ Beijing \\ Institute of Biotechnology, Beijing, \\ People's Republic of China; ${ }^{3}$ College \\ of Biological Engineering, HeNan \\ University of Technology, Beijing, \\ People's Republic of China \\ *These authors contributed equally \\ to this work
}

Background: Many reports have indicated that the intravenous administration of bevacizumab produces a number of systemic side effects. Therefore, we investigated the therapeutic effects of intratumoral bevacizumab administration using a glioma animal model.

Methods: The glioma cell lines U251 and U87 that carried luciferase were implanted into the brains of mice to develop glioma models. Glioma-bearing mice were treated with bevacizumab intravenously or intratumorally by Alzet micro-osmotic pumps, and the survival time of mice was monitored. Tumor volumes and location were observed by fluorescence imaging and histological analysis. Levels of microvessel marker, cancer stem cell marker as well as angiogenesis-, invasion-, and inflammation-related factors in tumors were examined by immunohistochemical staining.

Results: Mice treated with intratumoral low-dose bevacizumab had smaller tumor volumes, longer survival time, lower microvessel density, and fewer cancer stem cells as compared with untreated and intravenously treated mice. Furthermore, expression levels of inflammation-related factors increased signifiwhereas that of angiogenesis- and invasion-related factors decreased in intratumorally treated animals, compared with intravenously treated mice.

Conclusion: These results implied bevacizumab delivery by intratumoral injection via Alzet micro-osmotic pumps may be a more effective and safer protocol for treating gliomas.

Keywords: bevacizumab, anti-angiogenic, glioma cell line, intratumoral delivery, fluorescence imaging

\section{Introduction}

Gliomas [glioblastoma multiforme (GBM)] are highly vascularized tumors and their abundant capillary network may possibly be the main reason for their rapid growth and poor prognosis. A series of studies proved that the vascular endothelial growth factor (VEGF) and its associated pathways are of great importance in controlling the complex process of angiogenesis in GBMs. ${ }^{1-3}$ Therefore, bevacizumab (BEV) - a recombinant humanized monoclonal antibody that binds to human VEGF - has found widespread application in the treatment of malignant gliomas and is intravenously administered. $^{4-6}$

However, some studies have indicated that chronic high-dose BEV treatment results in serious systemic adverse events in patients. In addition, tumors eventually became resistant to the anti-angiogenesis therapy and adopt a more invasive growth pattern. ${ }^{7-9}$ Thus, the systemic administration of BEV has exhibited limited effects on glioma progression and, reduction of the BEV dose to physiological levels is desirable. Therefore, defining a delivery method at a lower dose with lesser systemic adverse events and good therapeutic effect is both necessary and important.
Correspondence: Zhi-Wen Zhang Department of Neurosurgery, The First Affiliated Hospital of PLA General Hospital, 5I Fucheng Road, Beijing 100048, People's Republic of China $\mathrm{Tel} / \mathrm{fax}+86106684835$ I Email zhangzw30I@I63.com

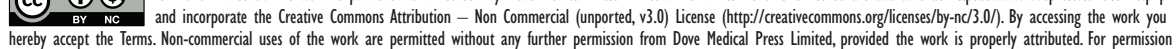

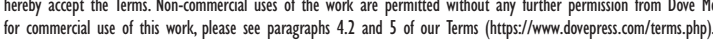


In the present study, we compared the therapeutic effect of intratumoral (IT) low-dose BEV treatment and intravenous (IV) high-dose BEV treatment in glioma-bearing animals with respect to the therapeutic effect and tumor-invasion capacity in glioma-bearing mice. The results showed that the administration of BEV by IT injection might be an effective protocol for treating gliomas by regulating the expression of inflammation- and invasion-related factors and inhibiting cancer stem cells [glioblastoma stem-like cells (GSCs)]; these findings help provide direction to future clinical trials of BEV using this novel drug-delivery method.

\section{Materials and methods}

\section{Human glioma cells stably expressing the luciferase reporter gene}

Lentiviral particles carrying the luciferase (luc) reporter gene were provided by the Qiang Zhao Xing Ye Company. U251 and U87 cells were purchased from the Cell Bank of the Chinese Academy of Sciences (Beijing, People's Republic of China) and transfected as previously described. ${ }^{10}$ Fortyeight hours after transfection, positive cells were sorted by fluorescence-activated cell sorting (FACS), according to the expression of green fluorescent protein (GFP).

\section{Mouse tumor model}

All animals used in this study were from the Laboratory Animal Center of the Academy of Military Medical Sciences of China (Beijing). All animal experiments were approved by the Ethical Committee of the Chinese PLA General Hospital (Beijing, People's Republic of China). The procedures in this study were conducted in accordance with the guidelines for the use of experimental animals from the National Institutes of Health. U251 or U87 glioma cells expressing the luc reporter gene $\left(1 \times 10^{6}\right.$ cells in $5 \mu \mathrm{L}$ PBS) were implanted into the brains of anesthetized athymic $\mathrm{nu} / \mathrm{nu}$ mice: a median incision of $\sim 1 \mathrm{~cm}$ was made, a burr hole was drilled into the skull, and cells were injected into the right striatum. The cell suspension in PBS $(5 \mu \mathrm{L})$ was injected at the rate of $1 \mu \mathrm{L} \mathrm{m^{-1 }}$ at a depth of $3 \mathrm{~mm}$. Seven days after tumor cell transplantation, the animals divided into three groups ( $n=10$ in each group): 1) untreated, 2) IT $\mathrm{BEV}$, and 3) IV BEV. In the IV treatment group, BEV was administered at a dose of $5 \mathrm{mg} / \mathrm{kg}$ via a tail-vein catheter once weekly (four injections in 28 days). The total BEV dose in the IV group was $20 \mathrm{mg} / \mathrm{kg}$ for 28 days. For the IT treatment group, an Alzet pump (Alzet, Cupertino, CA, USA) was implanted into each mouse as as previously described. ${ }^{11,12}$ Briefly, mice were anesthetized and the osmotic pump was placed into a subcutaneous pocket in the dorsal region. A cannula was implanted through the same hole used for glioma transplantation and was sealed with dental cement before it was connected to the pump. The pumps were filled up with vehicle (PBS), or BEV $\left(25 \mu \mathrm{g} \mu \mathrm{L}^{-1}\right)$ was delivered into the tumor continuously by direct IT delivery via an Alzet microosmotic pump at a total dose of $10 \mathrm{mg} / \mathrm{kg}$ for 28 days, which was half the dose that was used in the IV group for 28 days. Untreated tumor-bearing mice received PBS using the Alzet micro-osmotic pump as described earlier. After the treatment was completed ( 28 days), the animals were maintained without any treatment until they died spontaneously.

\section{Bioluminescence imaging in vivo}

Bioluminescence imaging (BLI) was used to assess tumor growth and calculate tumor volumes. Briefly, BLI was carried out in vivo using the NightOWL LB 983 in vivo Imaging System. Mice were anesthetized by an intraperitoneal injection of $10 \%$ chloral hydrate $(0.03 \mathrm{mg} / \mathrm{kg})$. D-luciferin (Sigma) dissolved in saline was then injected intraperitoneally at a dose of $100 \mathrm{mg} / \mathrm{kg}$ body weight. Immediately after the injection, a series of bioluminescent images was acquired with 2-minutes acquisition intervals for approximately 20 minutes, by which time, the luciferin had been washed out. The image with the peak BLI intensity was used for quantification in units of photon counts.

\section{Histology and immunohistochemistry}

Paraffin-embedded sections of brain tissues were deparaffinized by placing slides into three changes of xylene, followed by rehydration in graded ethanol. Then, hematoxylin staining was carried out and sections were rinsed with water until the slice became blue. Color separation was carried out using $70 \%$ alcohol, following by rinsing with water. The sections were stained with $1 \%$ eosin for 5-10 minutes, and then rinsed with distilled water. Thereafter, a gradient ethanol dehydration was undertaken. Finally, the sections were sealed with neutral resin, and observed by light microscopy.

For histological analysis, sections were incubated with anti-CD31, sex determining region Y-box 2 (SOX2), matrix metallopeptidase 9 (MMP-9), or cold-inducible RNAbinding protein (CIRP; Santa Cruz Biotechnology, CA, USA) antibodies at $4^{\circ} \mathrm{C}$ overnight, washed thrice with PBS, and incubated with the corresponding secondary antibodies for 1 hour. Images were acquired using a microscope. Microvessel density (MVD) was determined by immune-staining with CD31. The numbers of SOX2, MMP-9, or CIRP-positive cells per field were counted by ImageJ and divided by the 
number of total cells per field for normalization; the final outcome was defined as the percentage of positive cells/field and was the mean value calculated in 10 high-power microscopic fields.

\section{Statistical analysis}

All data were expressed as the mean \pm SD. Differences between the two groups were assessed by the unpaired Student's $t$-test, which is used to test differences in the data obtained from two groups of independent samples using SPSS version 19.0 (IBM Corporation, Armonk, NY, USA). Survival time was calculated from the date of tumor injection to the date of death, the log-rank test was used to determine statistical significance. A $p$-value $<0.05$ was considered indicative of statistical significance in the tests conducted.

\section{Results}

\section{Establishment of stable glioma cell lines carrying luc}

Lentivirus vectors carrying luc (Figure 1A) were transfected into U251 and U87 cells. The vectors and the resultant lentivirus carried GFP; therefore, infection efficiency in U251 and U87 cells was estimated by GFP expression. High-GFPexpression luc ${ }^{+} \mathrm{U} 251$ and U87 cells were sorted by FACS. After sorting, almost all of cells showed strong GFP signal (Figure 1B).

\section{IT BEV treatment at a lower dose could inhibit tumor growth}

U251-luc and U87-luc cells were implanted into the brains of NOD/SCID mice and tumor growth was monitored with
BLI. At Day 7 after cell implantation, animals were divided into three groups randomly (untreated, IT BEV, and IV $\mathrm{BEV}$ ) and, tumor growth was monitored with BLI every week (Figure 2). In U251 tumor-bearing animals, BLI signal intensity of the IT BEV-treated mice was the lowest among the three groups. The BLI signal intensity of IV-treated mice was lower than that of untreated mice. Moreover, results from the U87 tumor-bearing animals confirmed that the BLI signal intensity of the IT-treated group was significantly lower than that in the IV-treated or untreated groups. These results indicated that administration of BEV - either IV or IT - decreased tumor growth; however, IT BEV treatment was more efficient than IV BEV treatment.

\section{Outcome of histology was consistent with that of BLI}

Tumor tissues were obtained from animals at Day 28 after treatment. Representative photographs of hematoxylin \& eosin (HE)-stained brain sections from untreated, IV BEV, or IT BEV-treated mice are shown in Figure 3. Volumes of tumors in different groups were estimated on the images of HE-stained sections using Image-Pro PLUS software. For both U251 and U87 tumor-bearing animals, a significant decrease of tumor volumes was observed in mice from IV or IT BEV-treated groups as compared to untreated mice (U251: untreated vs IV, $p=0.0138$; untreated vs IT, $p=0.0008$; U87: untreated vs IV, $p=0.0025$; untreated vs IT, $p=0.0002$ ). More importantly, there was an apparent difference in tumor volumes between IV BEV and IT BEV-treated mice (U251: IT vs IV, $p=0.0012$; U87: IT vs IV $p=0.0088$ ). These data were in agreement with the results of BLI tracing.
A

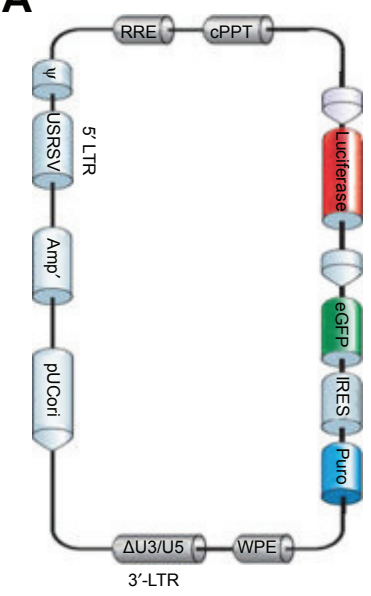

B
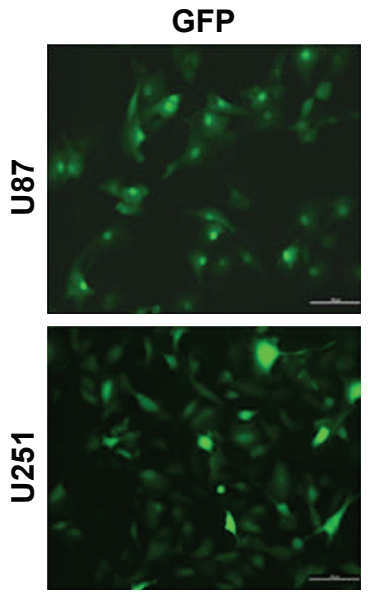

DAPI
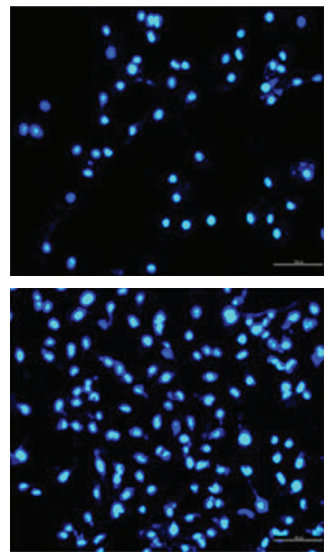

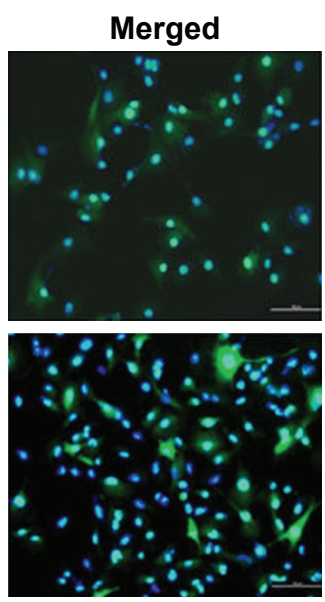

Figure I Establishment of stable glioma cell lines carrying luciferase. (A) The structure of lentivirus vectors carrying luciferase. (B) Luc ${ }^{+} \mathrm{U} 25 \mathrm{I}$ and $\mathrm{U} 87$ cells were sorted by fluorescence-activated cell sorting. After sorting, almost all of cells showed a strong green fluorescent protein (GFP) signal.

Note: Scale bar, $100 \mu \mathrm{m}$. 
A

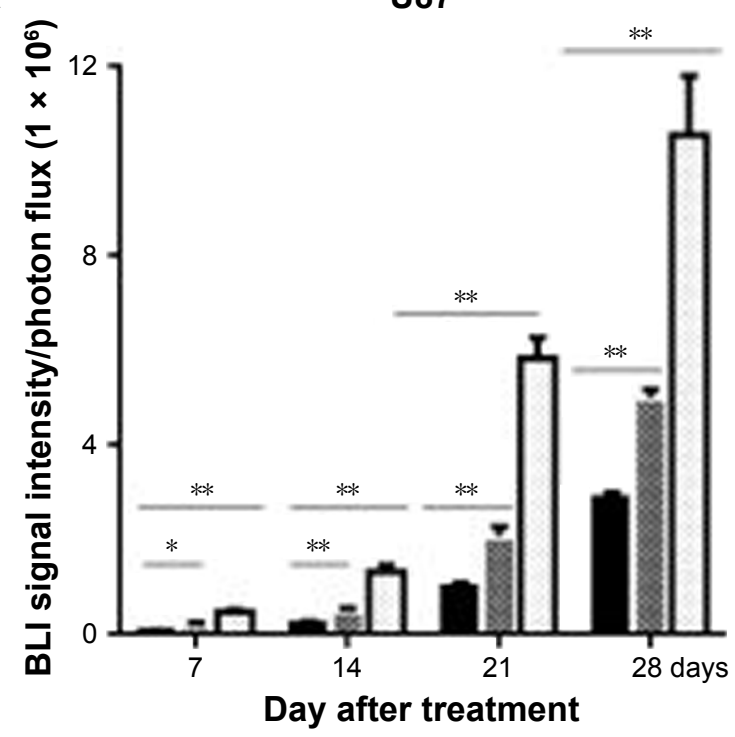

U251

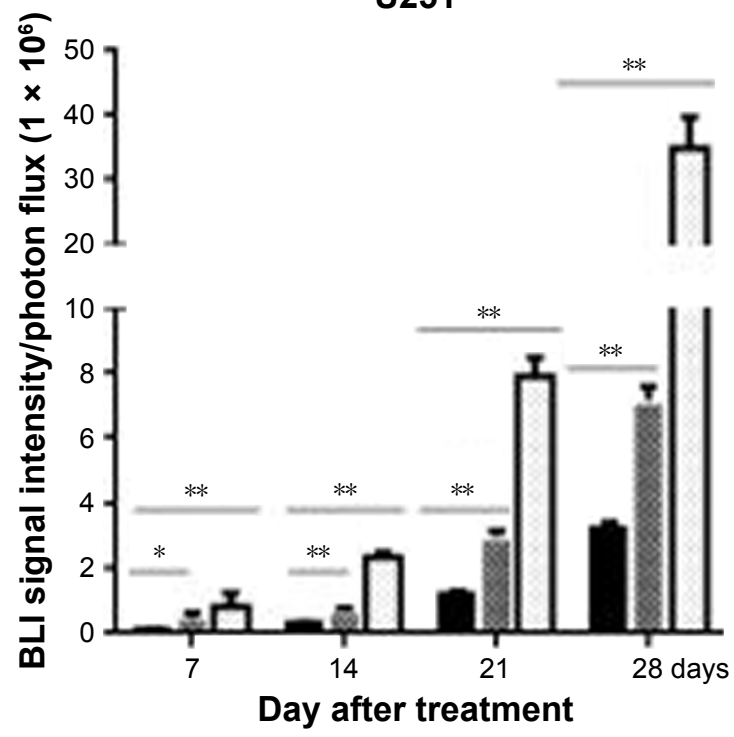

IT IV IV Untreated

B

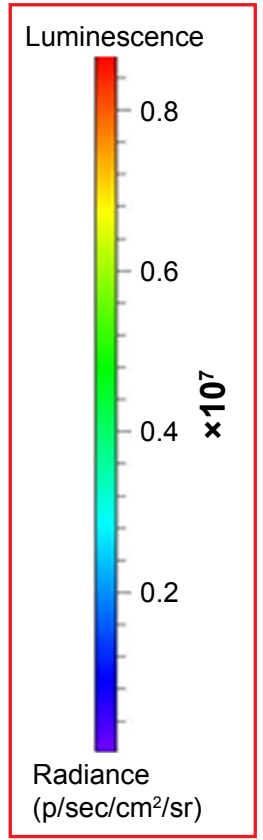

IT
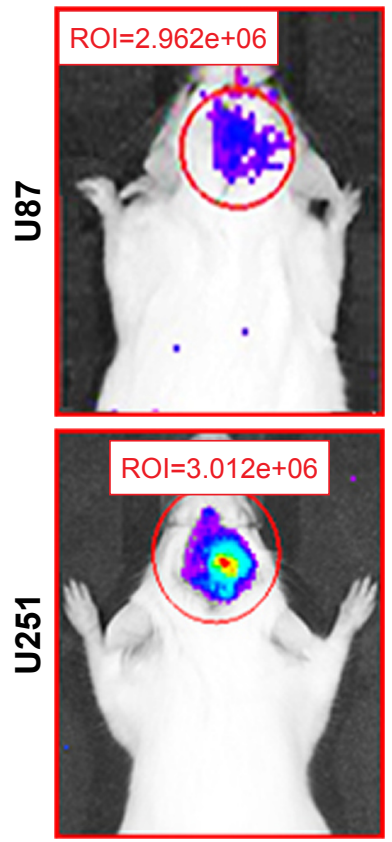

IV
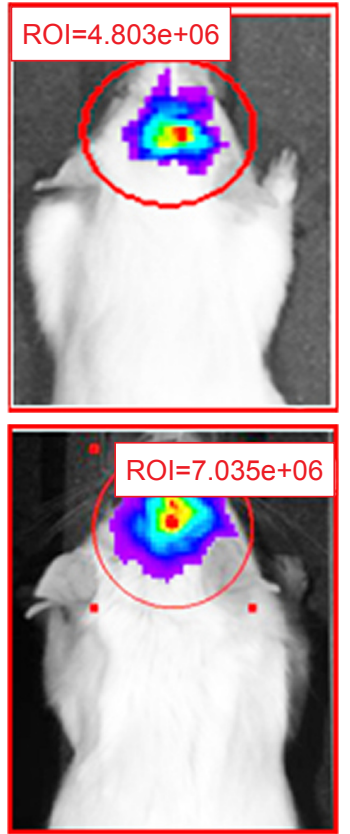

Untreated
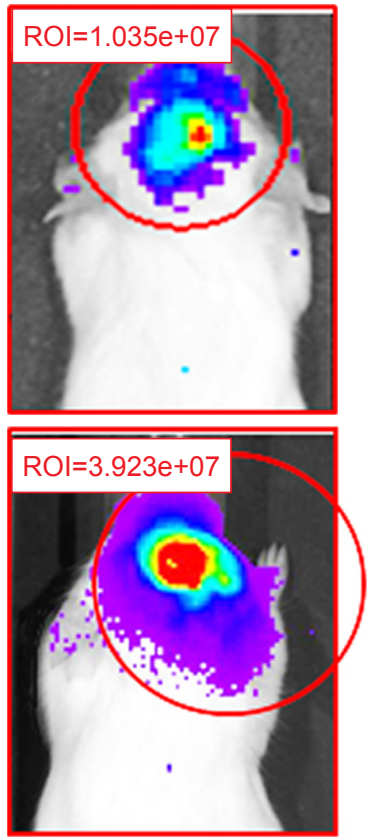

Figure 2 Luc expression in glioma in mice. (A) Statistical analyses for the BLI signal intensity of U87 and U25 I tumors every week. (B) The luminescence of U87 and U25I tumors in mice at Day 28 after BEV treatment.

Notes: Data represent the mean percentage of tumor area \pm SD $(n=10) . *_{p}<0.05, *^{*} p<0.01$.

Abbreviations: IT, intratumoral; IV, intravenousl; BEV, bevacizumab; BLI, Bioluminescence imaging.

\section{IT BEV treatment increased survival of mice}

To estimate the effect of delivery method on clinical outcome, we examined the survival of mice with glioma xenografts after IV or IT BEV treatment. Data from U251 tumor-bearing animals showed that BEV treatment significantly increased the survival time of glioma models. Moreover, the mice in the IT BEV treatment group survived longer than those in the IV treatment group, with a median survival of 40 days for the IT group, 27 days for the IV group, and 17 days for the untreated group (untreated vs IT, $p=0.0083$; IV vs IT, $p=0.0042$; Figure 4A).

Furthermore, we observed a significant prolongation of survival time in U87 tumor-bearing animals after IT BEV 


\section{A}
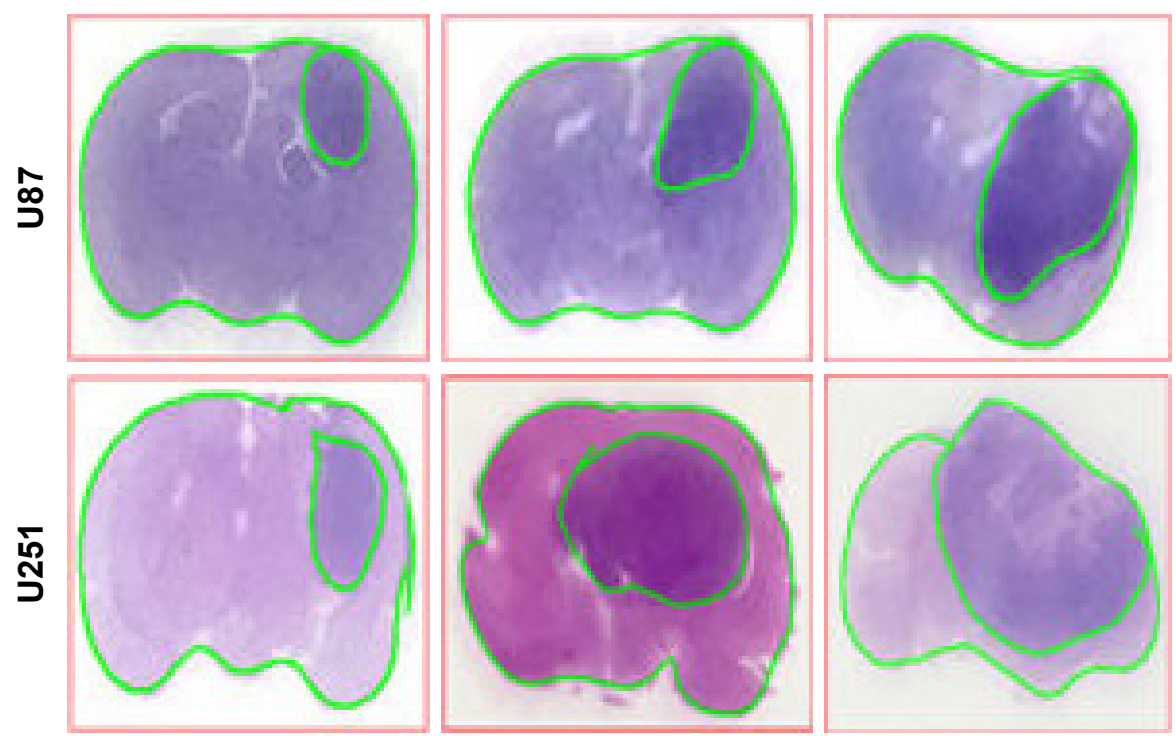

B

U87
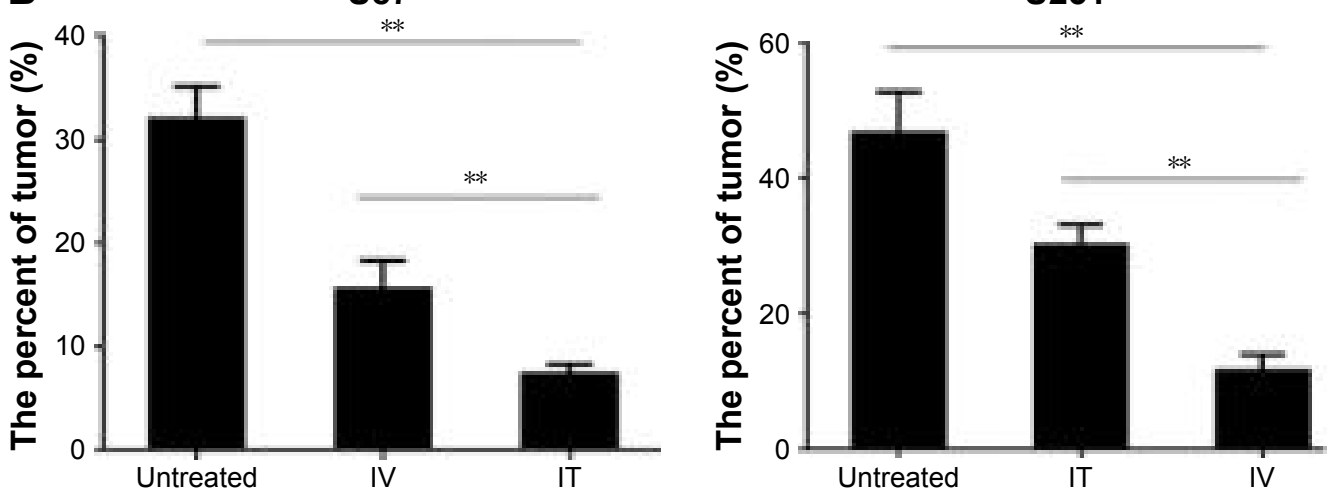

Figure 3 Volumes of tumors in different groups. (A) Histopathology of a brain section stained with hematoxylin and eosin. (B) Tumor volume was estimated using Image-Pro PLUS and expressed as a ratio of tumor area against the whole brain area.

Notes: Data represent the mean percentage of tumor area $\pm S D(n=10)$. $* * p<0.01$.

Abbreviations: IT, intratumoral; IV, intravenous.

treatment, with a median lifespan of 23 days for controls, 32 days for IV BEV-treated animals, and 45 days for IT BEV-treated animals (untreated vs IT, $p=0.0017$; IV vs IT, $p=0.0047$; Figure 4B).

A

U251

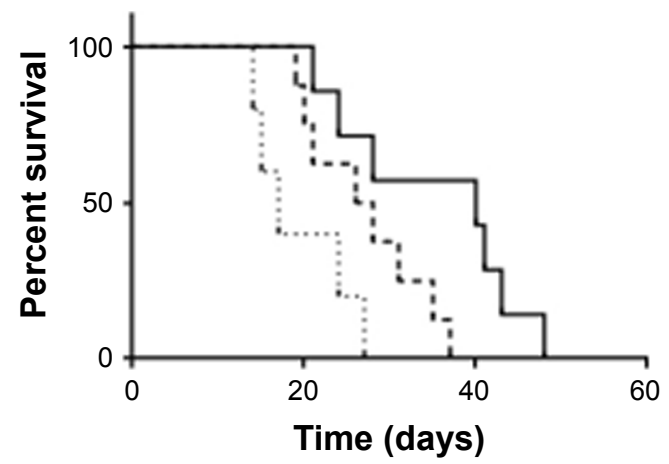

\section{IT BEV treatment decreased MVD}

To determine the potential mechanism, tumor tissues were obtained from animals at Day 28 after treatment. Previous studies reported that BEV treatment transiently normalized

B

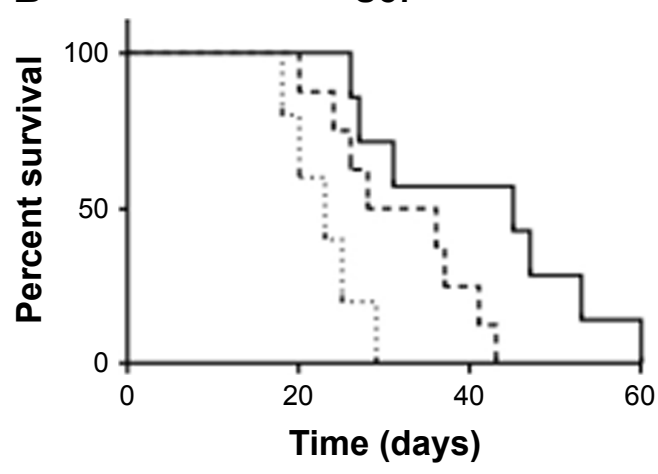

Figure 4 IT BEV treatment increased the survival of mice. The survival time of animals bearing U25I (A) or U87 (B) tumors was analyzed by Kaplan-Meier analysis. Abbreviations: IT, intratumoral; IV, intravenous. 

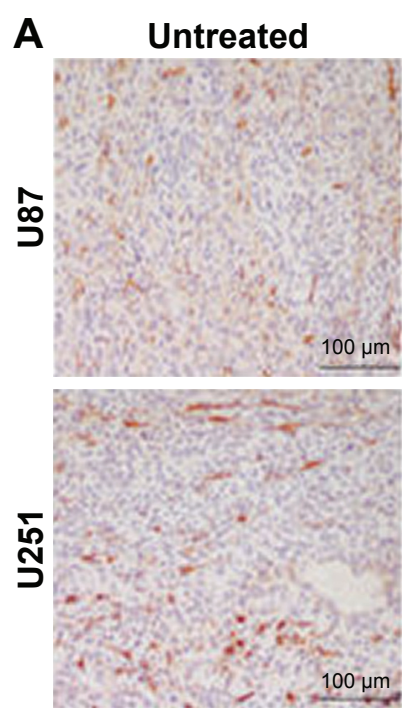

IT
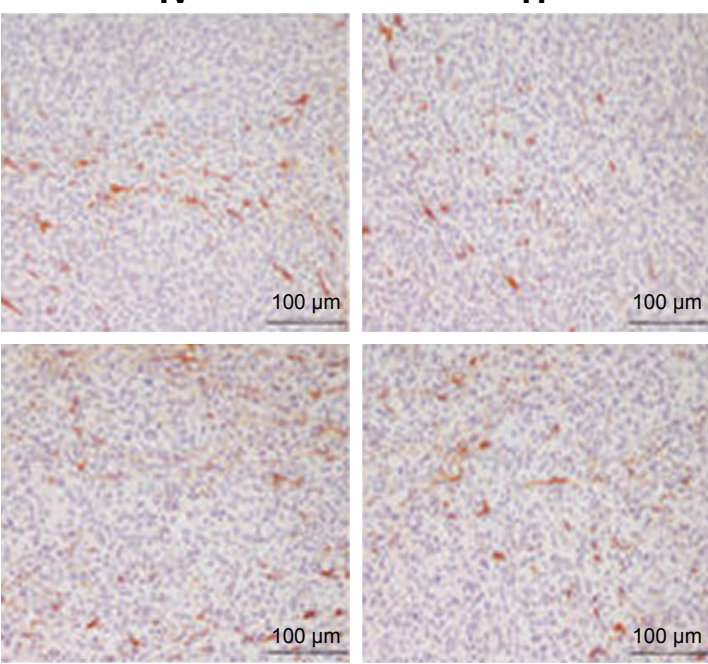

IV
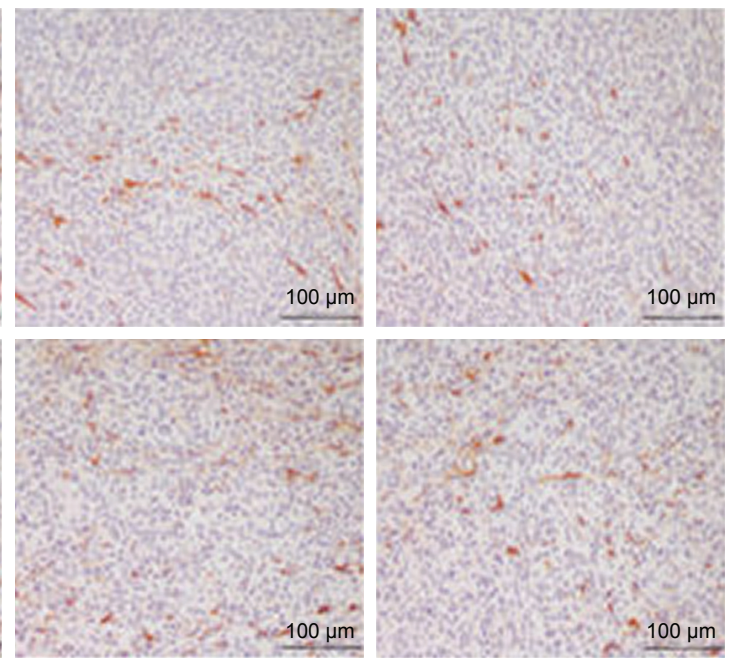

B
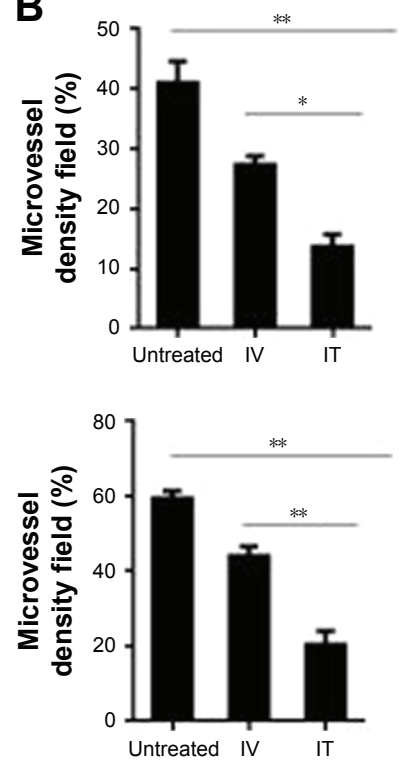

Figure 5 IT BEV treatment decreased the MVD. Tumor tissues from the different groups were immunostained for blood vessels using CD3I. (A) Histological specimens show fewer blood vessels in IT BEV-treated tumors than in untreated or IV BEV-treated specimens. Scale bar, $100 \mu \mathrm{m}$. (B) Statistical analysis for the relative number of microvessel density (MVD). CD3I-positive areas were regarded as vessels and determined by Imagej; vessels per field were counted as the MVD.

Notes: Data are represented as mean \pm SD $(n=10)$. $* p<0.05$; ** $p<0.01$.

Abbreviations: IT, intratumoral; IV, intravenousl; BEV, bevacizumab.

abnormal tumor blood vessels. In the present study, we analyzed the MVD of tumor by immunostaining for CD31 - a vessel-density marker to quantify the number of blood vessels (Figure 5). Not surprisingly, U251 tumors showed a marked reduction in the relative density of blood vessels in BEVtreated animals, compared with untreated controls $(p<0.01)$; moreover, the data showed that IT BEV significantly reduced the density of tumor blood vessels as compared with that in the IV group (approximately $50 \%$ reduction, $p<0.01$ ). Further, results from the U87 tumor-bearing animals showed approximately $40 \%$ reduction of blood vessels in the IT $\mathrm{BEV}$ group as compared with those in the IV BEV-treated group $(p<0.05)$.

\section{IT BEV treatment decreased cancer stem cells}

To determine whether the method of drug delivery had an effect on CSCs, tumor tissues from different groups were analyzed for SOX2 - the marker for CSCs. The results showed that the number of $\mathrm{SOX}^{+}$cells within the tumors of the BEV-treated groups was significant less than in the untreated group (U251: control vs IV, $p=0.04$; control vs IT, $p=0.0023$. U87: control vs IV, $p=0.02$; control vs IT, $p=0.0012$ ). More importantly, $\mathrm{SOX} 2^{+}$cells in the tumors of the IT group decreased significantly as compared to that of the IV group ( $p<0.002$; Figures 6 and 7 ).

\section{Invasive factors of tumors decreased after IT BEV treatment}

Previous studies have reported that BEV treatment affected the invasive pattern of glioblastoma in vivo and vitro. Here, we evaluated the level of invasion-related factor MMP-9 in tumors from the IT or IV groups by immunostaining. The results from both U251 and U87 cells showed that MMP-9 expression in the xenograft tumors of IT group was significantly lower than that of the IV-treated group (Figure 8).

\section{Inflammatory factors of tumors increased after IT BEV treatment}

Inflammatory responses are important to inhibit tumor progression; therefore, we tested whether there were differences in the inflammatory response as a result of the BEV delivery method. Tissues from different groups were analyzed for the expression of CIRP - an important inflammatory factor mediating brain injury (Figure 9). Our results showed significantly increased CIRP expression in tumors from IT BEV-treated mice compared with IV BEV-treated mice (for $\mathrm{U} 251, p=0.0097$; for U87 cells, $p=0.006$ ).

\section{Discussion}

BEV - a VEGF-neutralizing antibody - is the most developed and successful treatment for glioblastoma. Currently, systemic high-dose administration is the main delivery method of $\mathrm{BEV}$ in glioma. However, with widespread use of BEV, 

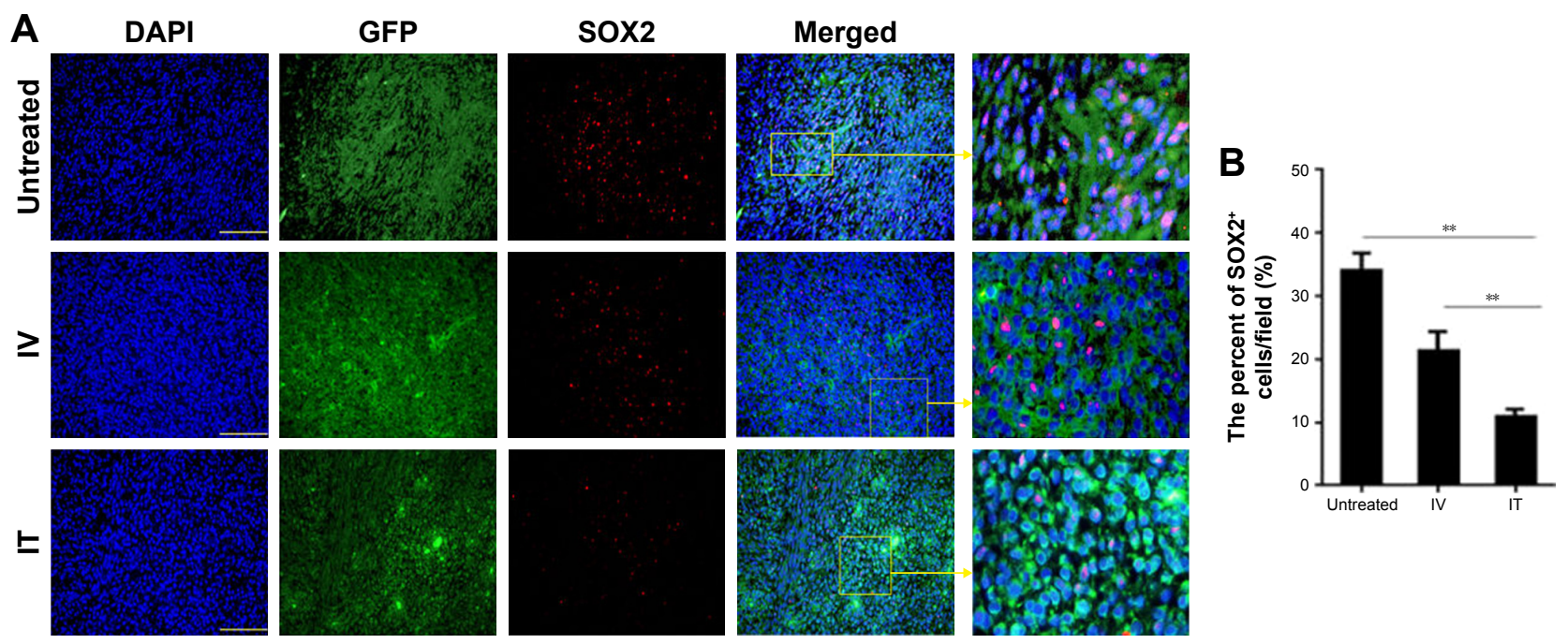

Figure $6 \mathrm{SOX} 2^{+}$cells within the tumors of U87 from different groups. Immunohistochemistry was conducted using SOX2 to determine the percentage of glioblastoma stemlike cells (GSCs) in the tumor xenografts of U87 cells. (A) Histological specimens show fewer GSCs in IT BEV-treated tumors than in untreated or IV BEV-treated specimens. Scale bar, $50 \mu \mathrm{m}$. (B) Statistical analysis of the percentage of SOX2+ cells within the tumors. Animals that received IT BEV had fewer SOX2 cells in the tumors than animals that were untreated or treated with IV BEV. The final outcome was calculated as a ratio (SOX2 positive cells/all cells in a field).

Notes: Data are represented as mean $\pm S D(n=10), * * p<0.01$.

Abbreviations: IT, intratumoral; IV, intravenousl; BEV, bevacizumab.

both inevitable and unexpected adverse vascular events have been observed. ${ }^{13-15}$ It is well known that VEGF is secreted by glioma cells and exists in the interstitial spaces of tumor cells. It interacts with VEGF receptors on endothelial cells of blood vessels and opens up the tumor-blood interface. ${ }^{16}$ Therefore, the location of BEV was critical, and we speculated that when BEV was directly delivered into the interstitial space, it could eliminate the VEGF secreted by glioma cells more effectively and the dose needed would be lower. To test this hypothesis, glioma animal models were treated with $\mathrm{BEV}$ $10 \mathrm{mg} / \mathrm{kg}$ intravenously or $5 \mathrm{mg} / \mathrm{kg}$ intratumorally (half the dose of the IV treatment) weekly. Then, we analyzed the effects of the BEV delivery method on tumor growth by in vivo bioluminescence imaging - a noninvasive laboratory and clinical imaging technique at the molecular level that can monitor the localization, proliferation, migration, and differentiation of cells in vivo. ${ }^{17}$ Herein, we found that luciferase activity in IT BEV-treated groups were much lower
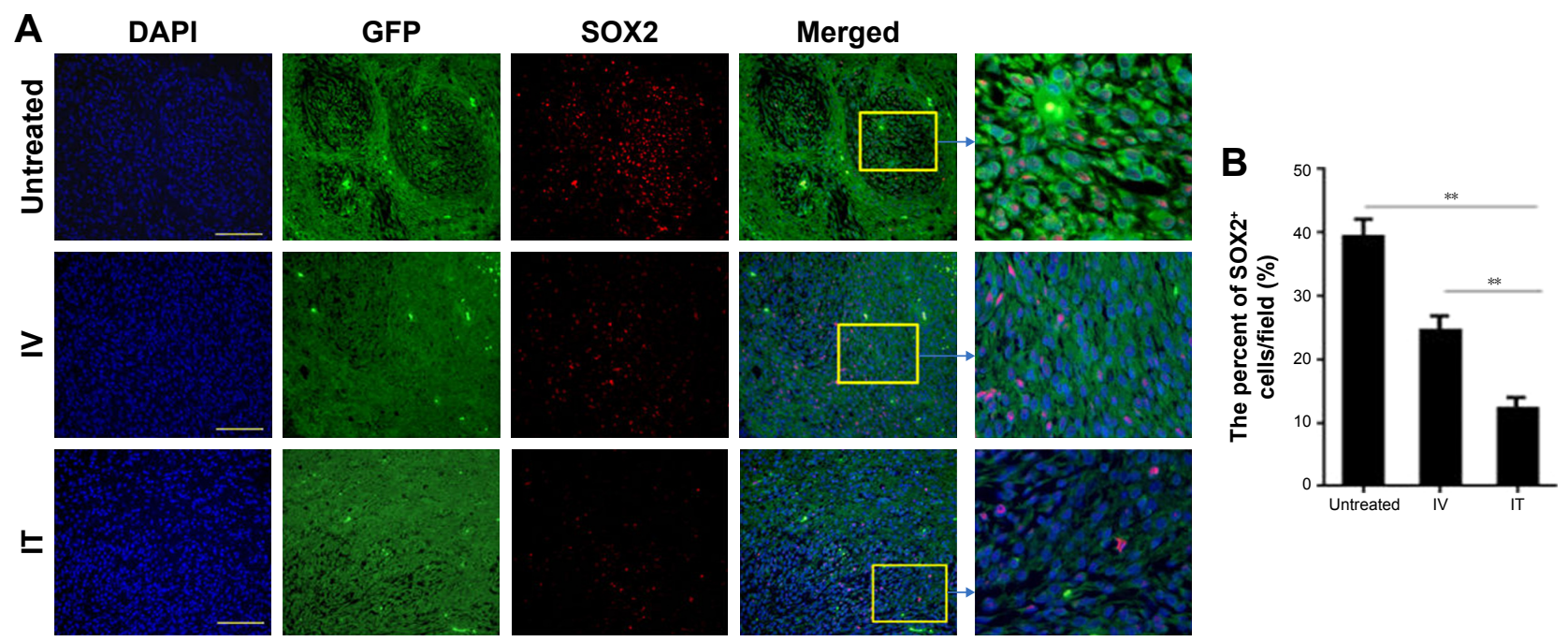

Figure $7 \mathrm{SOX} 2^{+}$cells within the tumors of U25I from different groups. Immunohistochemistry was conducted using SOX2 to determine the percentage of glioblastoma stem-like cells (GSCs) in the tumor xenografts of U87 cells. (A) Histological specimens show fewer GSCs in IT bevacizumab (BEV)-treated tumors than inuntreated or IV BEV-treated specimens. Scale bar, $50 \mu \mathrm{m}$. (B) Statistical analysis of the percentage of SOX $2^{+}$cells within the tumors. Animals that received IT BEV had fewer SOX2 ${ }^{+}$cells in the tumors than those that were untreated or treated with IV BEV. The final outcome was calculated as a ratio (SOX2 positive cells/all cells in a field).

Notes: Data are represented as mean \pm SD $(n=10),{ }^{* *} p<0.01$.

Abbreviations: IT, intratumoral; IV, intravenousl; BEV, bevacizumab. 
A

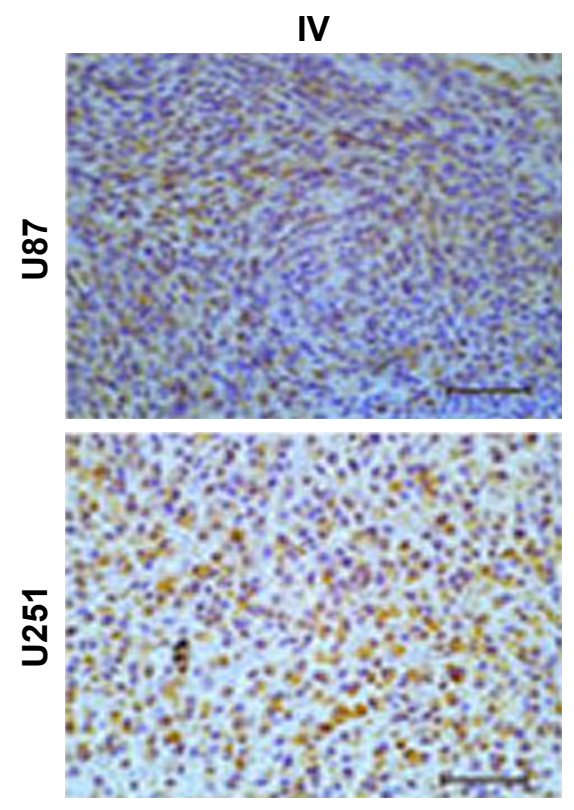

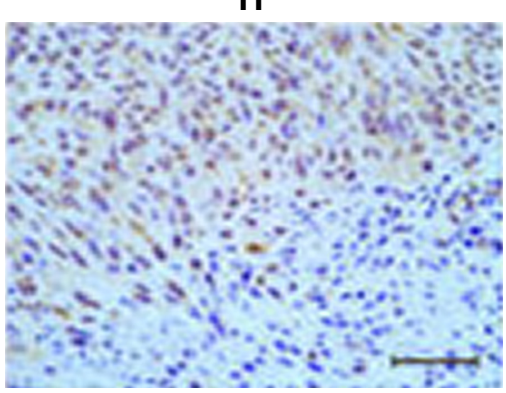

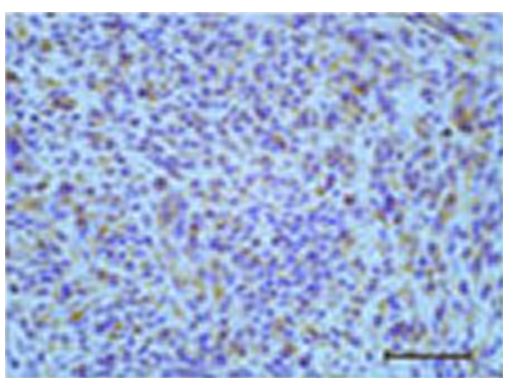

B
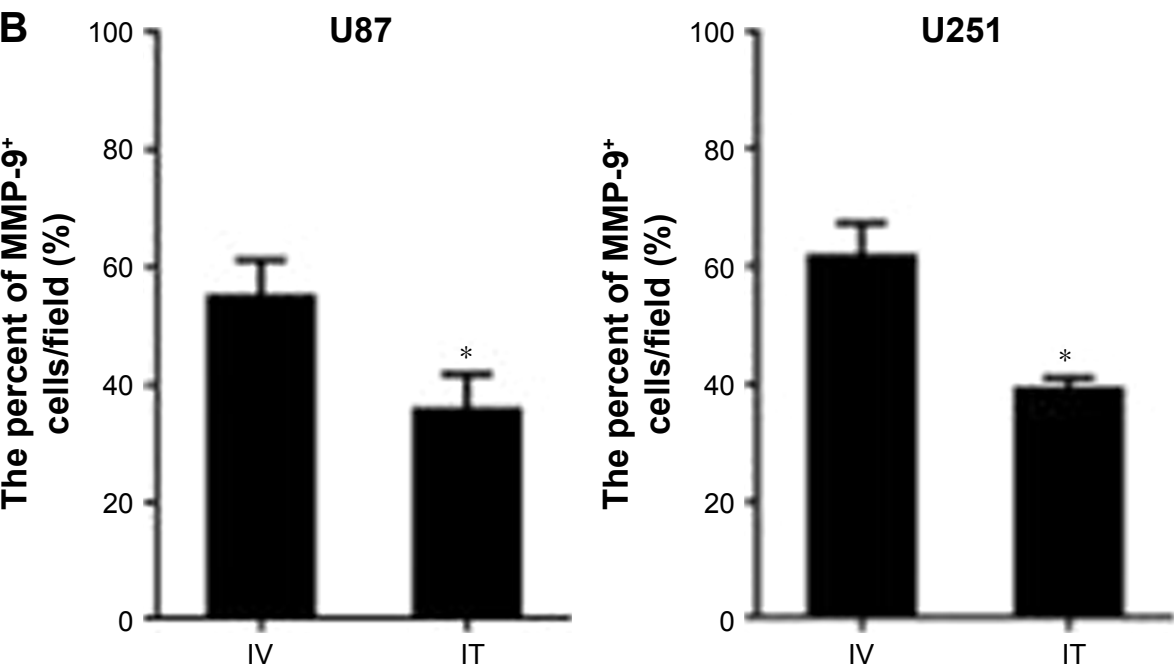

Figure 8 The expression of MMP-9 in tumors from different groups. Immunohistochemistry was conducted using MMP-9 in tumor xenografts. (A) Fewer MMP-9+ cells were observed in IT bevacizumab (BEV)-treated tumors as compared with IV BEV-treated specimens. Scale bar, $50 \mu \mathrm{m}$. (B) Statistical analysis of the percentage of MMP-9+ cells within the tumors. The final outcome was calculated as a ratio (MMP-9+ cells/all cells in a field).

Note: Data are represented as mean \pm SD $(n=10),{ }^{*} p<0.05$.

Abbreviations: IT, intratumoral; IV, intravenousl; BEV, bevacizumab.

than that in the IV BEV-treated and untreated groups, which proved that IT BEV treatment at a lower dose could inhibit tumor growth more efficiently than the IV BEV method. To further confirm the outcome of BLI, the brains of mice in different groups were isolated at Day 28 after BEV treatment. We found the results from histology were in accordance with those from BLI, which proved that BLI could offer a rapid and accurate means for studying tumor cell growth and response to therapy in animal models.

Next, we examined animal survival in different groups after BEV treatment. The results showed that the animal survival time of mice in the BEV-treated group was up to 3 times longer than in the control group. In addition, the mice treated with BEV intratumorally by Alzet micro-osmotic pumps survived longer than those treated systemically.

Glioblastoma displays remarkable cellular heterogeneity and differentiation hierarchy, because cancer cells contain GSCs that are able to self-renew, differentiate, vascularize, and become resistant to therapy. ${ }^{18,19}$ To interrogate the potential link between the drug-delivery method and tumor growth, we examined the expression pattern of the GSC marker (SOX2) in tumor specimens by immunofluorescence and found that SOX2 preferentially decreased after BEV treatment, especially after IT BEV treatment.

Clinical evidence has shown that anti-VEGF treatment increases tumor invasion in the brain, and the enhanced 
A

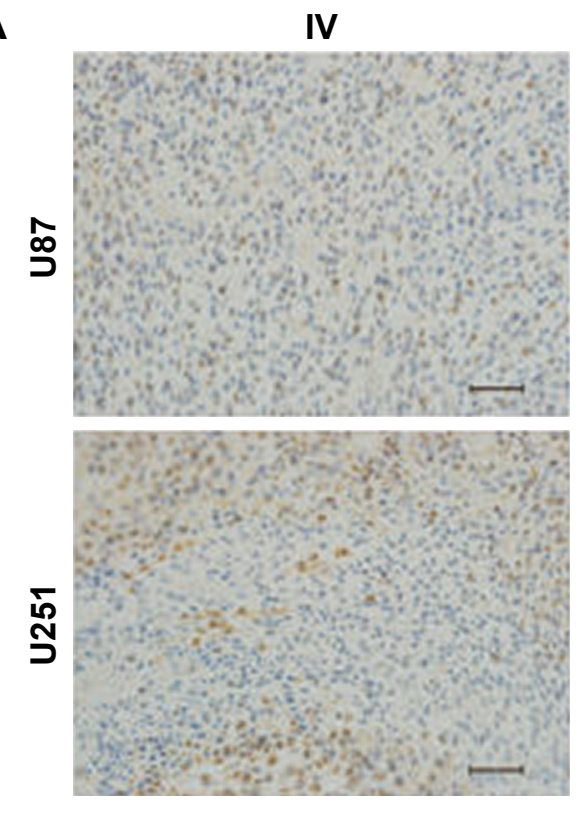

B

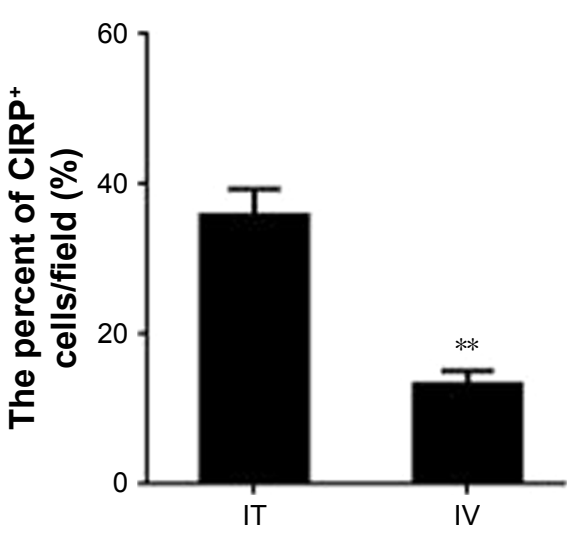

IT
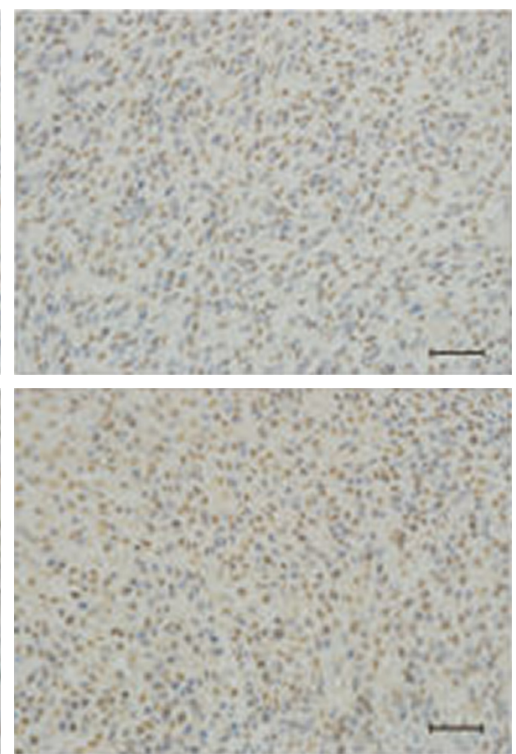

U251

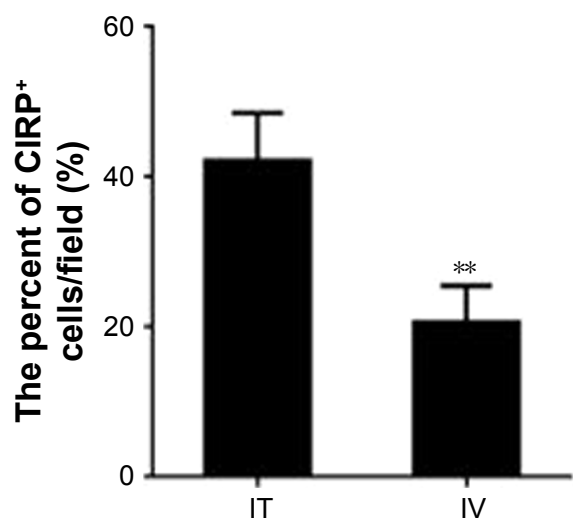

Figure 9 The expression of CIRP in tumors from different groups. Immunohistochemistry was conducted to determine the percentage of cold-inducible RNA-binding protein (CIRP)-positive cells in tumor xenografts. Scale bar, $50 \mu \mathrm{m}$. (A) Histological specimens show more CIRP ${ }^{+}$cells in IT, than in IV, BEV-treated tumors. (B) Statistical analysis of the percentage of $\mathrm{CIRP}^{+}$cells within the tumors. The final outcome was calculated as a ratio (CIRP+ cells/all cells in a field).

Notes: Data are represented as mean \pm SD $(n=10)$, ${ }^{* *} p<0.01$.

Abbreviations: IT, intratumoral; IV, intravenousl; BEV, bevacizumab.

invasion may be a result of the upregulation of enzymes which are responsible for the breakdown of extracellular matrix, such as MMPs. ${ }^{20-22}$ In the MMP family, MMP-9 is one of the most common metalloproteinases associated with neovascularization and tumor cell migration. ${ }^{23} \mathrm{Here}$, an expected anti-angiogenic effect was observed in both the IV and IT BEV-administration groups that were accompanied by a much reduced MVD at 4 weeks after drug treatment. Simultaneously however, strong expression of MMP-9 was found in tumors after BEV treatment. However, we observed a less invasive pattern of tumor growth in IT BEV-treated mice than in the IV groups with lower levels of MMP-9, which clearly indicated that IT BEV treatment at a low dose could alleviate invasion after long-term exposure to anti-VEGF therapy.
Inflammatory actions have been shown to play an important role in regulating the immune response to tumor, and proinflammatory factors are key to blocking tumor progression. ${ }^{24}$ Recent studies have revealed that extracellular CIRP is an important mediator in neuro-inflammation, which could induce release of tumor necrosis factor- $\alpha$ through activation of the NF- $\kappa \mathrm{B}$ pathway. ${ }^{25,26}$ Our data demonstrated a stronger expression of CIRP in tumors from the IT group, which implied the IT delivery of BEV may delay tumor growth by an intense inflammatory response.

Venous thromboembolism was one of the most popular side effects of IV BEV treatment; therefore, hemorheological parameters could be an important reference for the risk of thromboembolism. We measured the hemorheological 
characteristics of mice after BEV treatment and found that low-dose IT BEV treatment could decrease the risk of thromboembolism with a lower viscosity, when compared with IV treatment (data not shown).

In this study, untreated mice in whom the IT route was used were regarded as controls. For a more rationalized comparison, we examined the tumor volume and survival time in untreated mice in whom the IV route was used (tumorbearing mice received PBS intravenously) and found there were no significant differences between these two groups of untreated mice (Figure S1).

\section{Conclusion}

Localized BEV delivery by Alzet micro-osmotic pumps is more effective in reducing tumor size and tumor cell infiltration when compared with systemic administration. Thus, IT BEV delivery could be considered a good alternative to IV BEV administration. The identification of this fact paves the first step in the development of a novel delivery method to overcome the side effects of anti-angiogenic agents, and is worth investigating further.

\section{Acknowledgment}

This study was funded by the Special Scientific Research on Health Development of the Capital (grant no 2014-2-5021) and the National Natural Sciences Foundation of China (grant no 81472372).

\section{Author contributions}

Yu-Xiao Liu, Hui-Ru Zhang, and Wen-Jia Liu conducted the experiments. Zhi-Wen Zhang designed the study, analyzed and interpreted the data, and drafted the manuscript. All authors contributed toward data analysis, drafting and critically revising the paper and agree to be accountable for all aspects of the work. All authors have read and approved the final manuscript.

\section{Disclosure}

The authors report no conflicts of interest in this work.

\section{References}

1. Plate KH, Scholz A, Dumont DJ. Tumor angiogenesis and antiangiogenic therapy in malignant gliomas revisited. Acta Neuropathol. 2012;124(6):763-775.

2. Jain RK, di Tomaso E, Duda DG, Loeffler JS, Sorensen AG, Batchelor TT. Angiogenesis in brain tumours. Nat Rev Neurosci. 2007; 8(8):610-622.

3. Plate KH, Breier G, Weich HA, Risau W. Vascular endothelial growth factor is a potential tumour angiogenesis factor in human gliomas in vivo. Nature. 1992;359(6398):845-848.

4. Gutin PH, Iwamoto FM, Beal K, et al. Safety and efficacy of bevacizumab with hypofractionated stereotactic irradiation for recurrent malignant gliomas. Int J Radiat Oncol Biol Phys. 2009;75(1):156-163.
5. Lai A, Filka E, McGibbon B, et al. Phase II pilot study of bevacizumab in combination with temozolomide and regional radiation therapy for up-front treatment of patients with newly diagnosed glioblastoma multiforme: interim analysis of safety and tolerability. Int J Radiat Oncol Biol Phys. 2008;71(5):1372-1380.

6. Narayana A, Golfinos JG, Fischer I, et al. Feasibility of using bevacizumab with radiation therapy and temozolomide in newly diagnosed high-grade glioma. Int J Radiat Oncol Biol Phys. 2008;72(2):383-389.

7. Kabbinavar FF, Schulz J, McCleod M, et al. Addition of bevacizumab to bolus fluorouracil and leucovorin in first-line metastatic colorectal cancer: results of a randomized phase II trial. J Clin Oncol. 2005; 23(16):3697-3705

8. Sobolewska B, Grimmel C, Gatsiou A, et al. Different effects of ranibizumab and bevacizumab on platelet activation profile. Ophthalmologica. 2015;234(4):195-210.

9. Piao Y, Liang J, Holmes L, Henry V, Sulman E, de Groot JF. Acquired resistance to anti-VEGF therapy in glioblastoma is associated with a mesenchymal transition. Clin Cancer Res. 2013;19(16):4392-4403.

10. Liu YX, Dong X, Gong F, et al. Promotion of erythropoietic differentiation in hematopoietic stem cells by SOCS3 knock-down. PLoS One. 2015;10(8):e0135259.

11. Sartorius T, Hennige AM, Fritsche A, Häring HU. Sustained treatment with insulin detemir in mice alters brain activity and locomotion. PLoS One. 2016;11(9):e0162124.

12. Tweedie D, Rachmany L, Rubovitch V, et al. Exendin-4, a glucagonlike peptide-1 receptor agonist prevents mTBI-induced changes in hippocampus gene expression and memory deficits in mice. Exp Neurol. 2013;239:170-182.

13. Simonetti G, Trevisan E, Silvani A, et al. Safety of bevacizumab in patients with malignant gliomas: a systematic review. Neurol Sci. 2014; 35(1):83-89.

14. Lucio-Eterovic AK, Piao Y, de Groot JF. Mediators of glioblastoma resistance and invasion during antivascular endothelial growth factor therapy. 2009;15(14):4589-4599.

15. Knizetova P, Ehrmann J, Hlobilkova A, et al. Autocrine regulation of glioblastoma cell cycle progression, viability and radioresistance through the VEGF-VEGFR2 (KDR) interplay. Cell Cycle. 2008;7(16): 2553-2561.

16. Hoeben A, Landuyt B, Highley MS, Wildiers H, Van Oosterom AT, De Bruijn EA. Vascular endothelial growth factor and angiogenesis. Pharmacol Rev. 2004;56(4):549-580.

17. Cao J, Li X, Chang N, et al. Dual-modular molecular imaging to trace transplanted bone mesenchymal stromal cells in an acute myocardial infarction model. Cytotherapy. 2015;17(10):1365-1373.

18. Najbauer J, Kraljik N, Németh P. Glioma stem cells: markers, hallmarks and therapeutic targeting by metformin. Pathol Oncol Res. 2014;20(4): 789-797.

19. Bao S, Wu Q, McLendon RE, et al. Glioma stem cells promote radioresistance by preferential activation of the DNA damage response. Nature. 2006;444(7120):756-760.

20. Egeblad M, Werb Z. New functions for the matrix metalloproteinases in cancer progression. Nat Rev Cancer. 2002;2(3):161-174.

21. Mott JD, Werb Z. Regulation of matrix biology by matrix metalloproteinases. Curr Opin Cell Biol. 2004;16(5):558-564.

22. Page-McCaw A, Ewald AJ, Werb Z. Matrix metalloproteinases and the regulation of tissue remodelling. Nat Rev Mol Cell Biol. 2007;8(3): 221-233.

23. Duffy MJ, McGowan PM, Gallagher WM. Cancer invasion and metastasis: changing views. J Pathol. 2008;214(3):283-293.

24. Goldszmid RS, Dzutsev A, Trinchieri G. Host immune response to infection and cancer: unexpected commonalities. Cell Host Microbe. 2014;15(3):295-305.

25. Qiang X, Yang WL, Wu R, et al. Cold-inducible RNA-binding protein (CIRP) triggers inflammatory responses in hemorrhagic shock and sepsis. Nat Med. 2013;19(11):1489-1495.

26. Zhou M, Yang WL, Ji Y, Qiang X, Wang P. Cold-inducible RNAbinding protein mediates neuroinflammation in cerebral ischemia. Biochim Biophys Acta. 2014;1840(7):2253-2261. 


\section{Supplementary material}

A

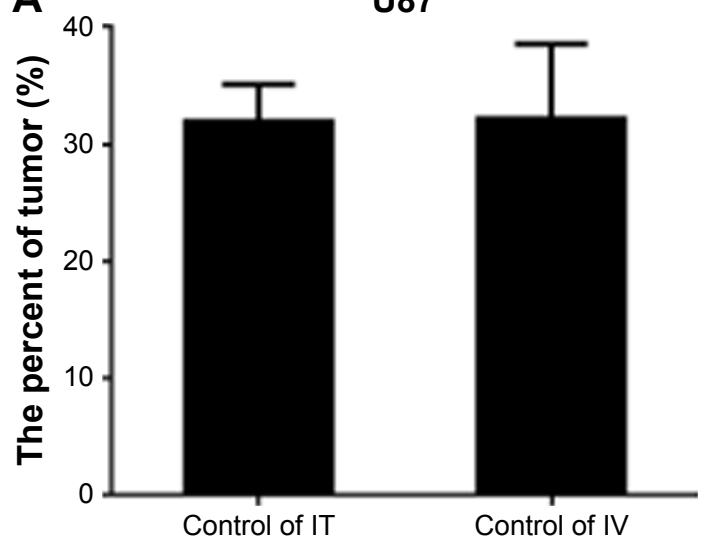

B

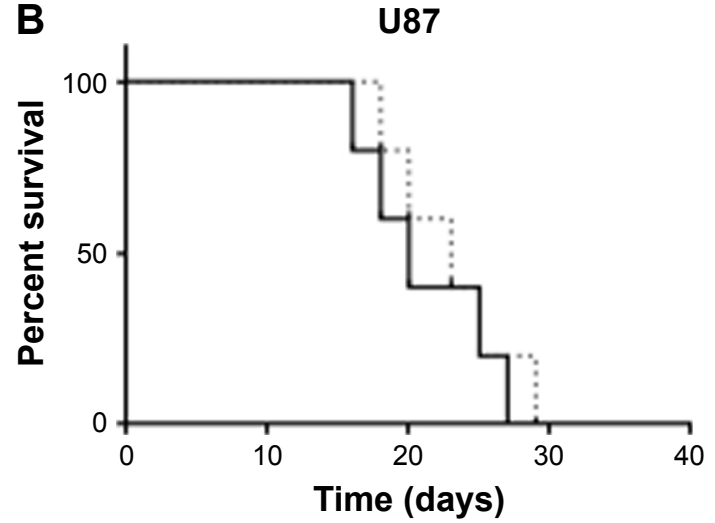

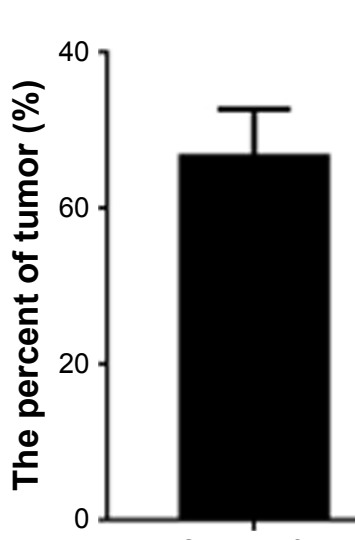

U251

Control of IT

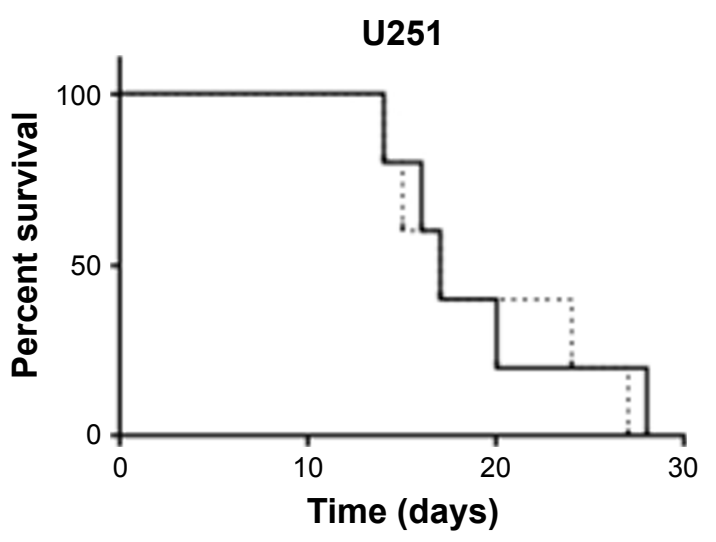

.... Control of IT $\quad$ 工 Control of IV

Figure SI Comparison of untreated IV and IT groups. (A) The survival time of untreated animals bearing U25I or U87 tumors was analyzed by Kaplan-Meier analysis. (B) Tumor volumes in untreated U25I or U87 groups were estimated using Image-Pro PLUS software and expressed as the ratio of the tumor area/the whole brain area. Note: Data represent the mean percentage of tumor area \pm SD $(n=10)$.

Abbreviations: IT, intratumoral; IV, intravenously.

\section{Publish your work in this journal}

OncoTargets and Therapy is an international, peer-reviewed, open access journal focusing on the pathological basis of all cancers, potential targets for therapy and treatment protocols employed to improve the management of cancer patients. The journal also focuses on the impact of management programs and new therapeutic agents and protocols on
Dovepress

patient perspectives such as quality of life, adherence and satisfaction. The manuscript management system is completely online and includes a very quick and fair peer-review system, which is all easy to use. Visit http://www.dovepress.com/testimonials.php to read real quotes from published authors. 